\title{
Brain Content of Cobalamin and Its Binders in Elderly Subjects
}

\author{
Masami InADA, Masanori ToYoshimA, and Masakuni KAMEYAMA \\ Department of Geriatrics, Kyoto University Hospital, \\ Shogoin-kawaramachi, Sakyo-ku, Kyoto 606, Japan
}

(Received December 11, 1981)

\begin{abstract}
Summary The biological aspects of cobalamin were studied in autopsy brains of eight elderly subjects who had lacked marked neurologic symptoms. Separation of the gray and white matter of the frontal and temporal lobes, and subsequently, subcellular fractionation of each matter were attempted. The contents of cobalamin and its binders and cobalamin concentrations were measured in each subcellular fraction and whole homogenate.

The contents of cobalamin were in the ranges of 50 to $60 \mathrm{ng} / \mathrm{g}$ wet tissue. No marked differences in contents were found between the frontal and temporal lobes. The mitochondria-rich fraction contained more cobalamin than did the microsomal fraction.

Two binders were found; a large-sized and a small-sized. The molecular weight of the larger binder was $c a$. 120,000, coinciding with the elution region of transcobalamin I, and the molecular weight of the smaller binder was 40,000 , coinciding with the elution region of transcobalamin II, in gel filtration respectively. More of the larger binder than the smaller was found in the mitochondria-rich and microsomal fractions.

These data are useful for the study of various aspects of cobalamin in brains with various types of neurologic pathology or aging changes.

Key Words cobalamin, cobalamin binders, frontal lobe, temporal lobe, gray matter, white matter, subcellular fraction, brain, mitochondria, elderly
\end{abstract}

Cobalamin levels and cobalamin binding protein (binders) have been extensively studied in the sera of patients with various diseases $(1,2)$. Studies on cobalamin metabolism in various organs, tissues, and cells is still limited although it elucidating aspects of metabolism in human tissues (3-5). Information concerning cobalamin metabolism in various organs, tissues, and cells is still limited although it has lately been increasing.

Combined degeneration of the spinal cord in pernicious anemia and peripheral

1 稲田雅美, 豊島正憲, 亀山正邦 
neuropathy due to diabetes mellitus are considerably improved by parenteral administration of cobalamin if given in an early stage, especially in the active form. From these clinical findings, it is conceivable that large amounts of cobalamin are present in the nervous tissues and that cobalamin is necessary for maintaining normal functions of the tissues. However, studies on the cobalamin present in the human nervous system are sparse in spite of the comparatively close relationship between the nervous system and cobalamin.

Therefore, studies were made on cobalamin in human autopsy brains as a control for elderly subjects in this paper. In particular, regional localization, cytoarchitectural and subcellular distribution of cobalamin in these brains were studied in detail.

\section{MATERIALS AND METHODS}

Radioactive cobalamin. $\left[{ }^{57} \mathrm{Co}\right.$ cyanocobalamin (CT-2, approximately $100 \mu \mathrm{Ci}$ / $\mu \mathrm{g})$ was purchased from the Radiochemical Centre, Amersham, England. Radioactive cobalamin was used for the separation of binders and for measurement of their unsaturated binding capacity.

Autopsy brains. Human brains were obtained at autopsy from eight cases who had not had significant cerebral disorders. The diagnoses had consisted of cardiac infarction, pneumonia, hypertension, neurosyphilis, rupture of aneurysm, cardiac insufficiency, and pulmonary emphysema. The subjects had not been given any preparations containing vitamin $\mathrm{B}_{12}$, either orally or parenterally. They consisted of 5 males and 3 females of mean age $79.8 \pm 6.7$ years. The brains were obtained one to fifteen hours after death. The frontal and temporal lobes were deep-frozen at $-30^{\circ} \mathrm{C}$ until used in the experiments. The gray and white matter were separated carefully in thin sections of each brain tissue, from the frontal and temporal lobes, respectively.

Separation of subcellular fractions. Several pieces of each tissue were homogenized in $0.32 \mathrm{M}$ sucrose Tris-EDTA buffer, $\mathrm{pH} 7.8$, in a Teflon homogenizer immersed in ice-cold water. The whole homogenate was centrifuged to remove nuclei and cell debris by low-speed sedimentation.

The supernatant was used to further separate subcellular fractions by modifications of Whittaker's method (6) as shown in Fig. 1.

Assay for cobalamin contents. Total cobalamin contents in whole homogenates and subcellular fractions were measured using a radio-competitive method.

For the assay of cobalamin, homogenates of tissues were treated by heating with a cyanide-containing glutamate buffer in boiling water. Cobalamin concentrations in the extracts from the tissues were estimated by using assay kits of vitamin $\mathrm{B}_{12}$ (Phadebas, Pharmacia Diagnostics, Sweden), in which cobalamin was adsorbed onto the solid phase of intrinsic factor. Cobalamin concentrations were calculated, based on standard curves. When there was considerable turbidity in the sample, it was pretreated in the following manner. Cobalamin was extracted by 


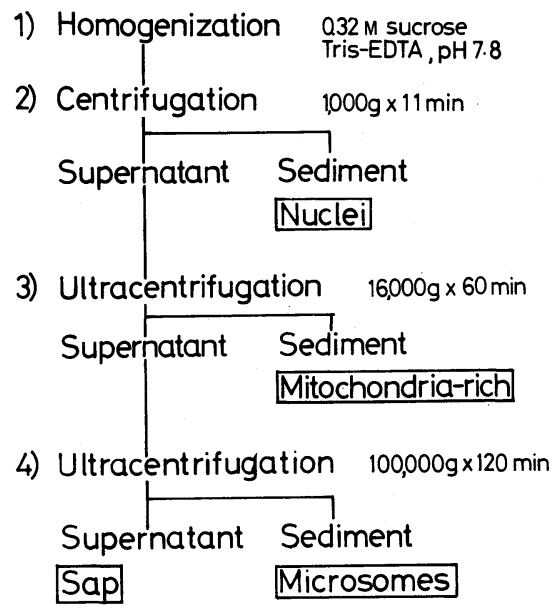

Fig. 1. Procedure of separation of the subcellular fractions.

heating the tissue homogenate in $80 \%$ ethanol at $80^{\circ} \mathrm{C}$ for one hour. After cooling, the extract was filtered through a filter paper. The filtrate was concentrated from a starting volume of $15.0 \mathrm{ml}$ to a final volume of $2.0 \mathrm{ml}$ under low atmospheric pressure. The concentrate was used as cobalamin solution for the assay of cobalamin.

Separation of cobalamin-binding proteins (binders). Each subcellular fraction was divided into two portions; one half was used for unsaturated binding capacity and the other for endogenous cobalamin.

Cobalamin binders were separated from subcellular fractions of the brains by using a $2 \times 28 \mathrm{~cm}$ column of Sephadex G 200 (Pharmacia, Sweden). Prior to application for gel filtration, subcellular fractions were presolubilized by adding Triton solution in a final concentration of $0.1 \%$. Subsequently, the solution containing $150 \mathrm{pg}$ of the $\left[{ }^{57} \mathrm{Co}\right]$ cyanocobalamin was added to the pretreated fractions to saturate the unsaturated binding capacity followed by incubation for $20 \mathrm{hr}$ at $4^{\circ} \mathrm{C}$. Immediately before the application, potassium hydroxide solution was added to the fraction to a final concentration of $0.02 \mathrm{~N}$ to completely dissociate the larger and smaller binders. A half-molar solution of sodium chloride was used for elution with a fraction volume of $5 \mathrm{ml}$ and flow rate of $20 \mathrm{ml} / \mathrm{hr}$.

Degree of saturation and endogenous cobalamin in the binders. The unsaturated binding capacity of the binder was calculated from the eluted radioactive peaks of ${ }^{57} \mathrm{Co}$ obtained by the filtration. One-half of the remaining sample was applied to the gel column without radioactive cobalamin to measure the endogenous cobalamin. The eluate showing the radioactive peak was used for measuring endogenous cobalamin. 


\section{RESULTS}

\section{Subcellular fractionation}

The purity of each subcellular fraction was assessed by electronmicroscopy. The mitochondrial fraction was found to be rich in mitochondria with minimal contamination. Therefore, the mitochondria-rich or mitochondria-containing fraction was used as the mitochondrial fraction in this study. The mitochondrial fraction was, therefore, found to be partially but not absolutely purified.

\section{Subcellular distribution of cobalamin in human brain}

The cobalamin contents of the whole homogenates were in the range of 50.0 to $60.0 \mathrm{ng} / \mathrm{g}$ of wet tissue. The contents of cobalamin were not significantly different between the frontal and temporal lobes. Between the gray and white matter of each lobe, there were no significant differences in cobalamin concentration.

The cobalamin content of the cytosol was the largest of the subcellular fractions.
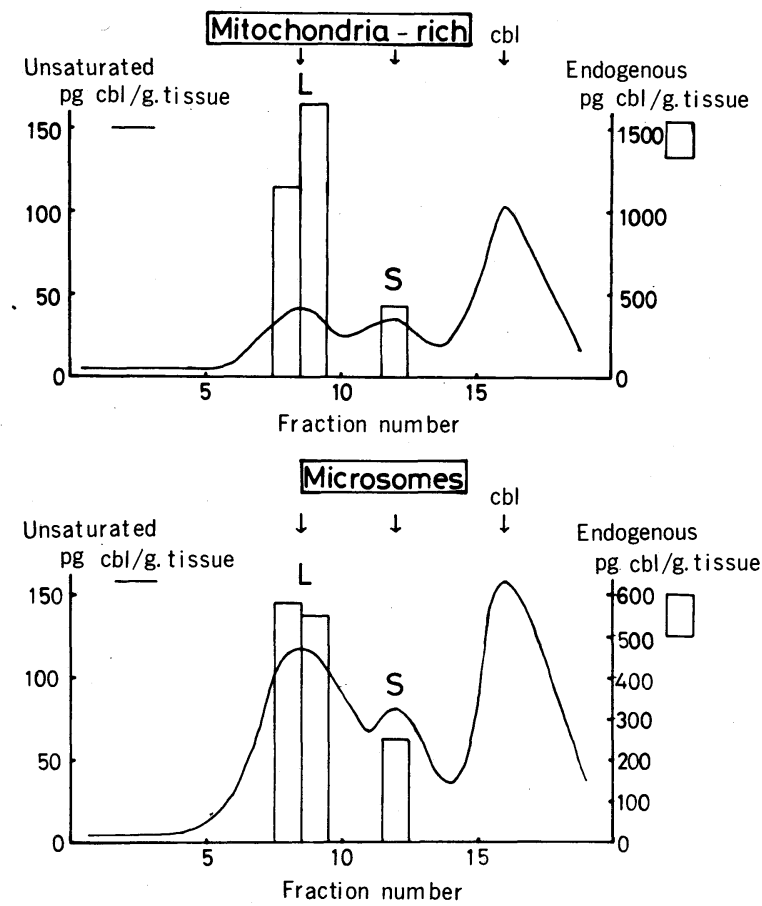

Fig. 2. Separation of cobalamin binders by gel filtration. Upper and lower figures show chromatographic patterns of the mitochondria-rich and microsomal fractions in the human brain, respectively. Solid curves represent radioactivities eluted and the columns indicate amounts of endogenous cobalamin bound to large (L) and small (S) binders. Each column corresponds with elution tube No. 8, No. 9, and No. 12, respectively. 


\section{Cobalamin binders}

In the filtration chromatogram, three radioactive peaks appeared, the first two peaks revealing unsaturated binding capacities of two binders; large and small. The first small peak coincided with the elution site of transcobalamin I, which has a molecular weight of $c a$ : 120,000 under the same conditions. The second peak appeared at an elution site similar to that of transcobalamin II with the molecular weight of 40,000. The last indicated unbound cobalamin. These eluates of larger and smaller molecular weights indicated the larger and smaller binders of cobalamin, respectively (Fig. 2).

Two binders were found in the human brains investigated. The larger binder and the smaller binder were almost saturated with endogenous cobalamin, as shown in the figure. The chromatographic patterns of the gel filtration did not show significant differences between the mitochondria-rich and microsomal fractions in the gray and white matter of the temporal and frontal lobes. More of the larger than of the smaller binder was seen in the mitochondria-rich and microsomal fractions. A greater amount of cobalamin binder was found in the mitochondria-rich fraction than in the microsomal fraction. No differences were found between the frontal and temporal lobes, in which no markedly clinical and pathological abnormalities were found at autopsy.

Table 1. Cobalamin contents of the whole homogenates and the subcellular fractions of the gray and white matter of human frontal and temporal lobes. Each figure shows mean $\pm \mathrm{SD}$ expressed as $\mathrm{ng} / \mathrm{g}$ wet tissues.

\begin{tabular}{llcccc}
\hline & & $\begin{array}{c}\text { Whole } \\
\text { homogenate }\end{array}$ & $\begin{array}{c}\text { Mitochondria- } \\
\text { rich }\end{array}$ & Microsomes & Cytosol \\
\hline \multirow{2}{*}{ Frontal } & Gray matter & $58.7 \pm 27.2$ & $7.3 \pm 2.4$ & $2.7 \pm 1.0$ & $17.8 \pm 5.6$ \\
& White matter & $66.2 \pm 11.5$ & $7.4 \pm 4.9$ & $2.7 \pm 1.6$ & $12.5 \pm 4.6$ \\
\hline \multirow{2}{*}{ Temporal } & Gray matter & $50.3 \pm 20.0$ & $11.1 \pm 5.7$ & $3.6 \pm 1.9$ & $26.2 \pm 10.3$ \\
& White matter & $52.2 \pm 15.4$ & $6.8 \pm 2.5$ & $2.6 \pm 1.2$ & $11.9 \pm 3.9$ \\
\hline
\end{tabular}

Table 2. Cobalamin binders in subcellular fractions. Each figure is expressed as $\mathrm{ng} \mathrm{cbl}$ binding/g wet tissue.

\begin{tabular}{clcc}
\hline & & $\begin{array}{c}\text { Mitochondria-rich } \\
\text { fractions }\end{array}$ & $\begin{array}{c}\text { Microsomal } \\
\text { fractions }\end{array}$ \\
\hline $\begin{array}{c}\text { Large-sized } \\
\text { binding protein }\end{array}$ & Frontal & $1.8 \pm 1.2$ & $1.0 \pm 0.5$ \\
& Temporal & $1.8 \pm 0.7$ & $1.0 \pm 0.5$ \\
\hline Small-sized & Frontal & $0.4 \pm 0.1$ & $0.3 \pm 0.1$ \\
binding protein & Temporal & $0.4 \pm 0.2$ & $0.3 \pm 0.1$ \\
\hline
\end{tabular}

Vol. 28, No. 4, 1982 


\section{DISCUSSION}

In the human brain, the total contents of cobalamin were found to be 50 to $60 \mathrm{ng} / \mathrm{g}$ of wet tissue. These values were lower than those given in one report (7), but were higher than those in another $(8)$. These levels showed an unexpectedly higher content relative to other human organs. Next to the liver and the kidney which have the highest concentrations, the brain contains considerably high concentrations of cobalamin. This finding indicated that cobalamin is readily transported into the brain and that cobalamin plays an important role in the brain.

As for distribution, cobalamin seems to be almost uniformly distributed throughout the brain. Between the gray and white matter or between the frontal and the temporal lobes, there were no gross differences in cobalamin distribution. Cobalamin seems to be highly concentrated in the pituitary of dogs (9). There are several anatomical structures showing exceptionally high contents of cobalamin in the human brain on comparison of the gray and white matter. Such structures are the median eminence of the hypothalamus, the pineal gland, and the line of attachment of the choroid plexus, which are excluded from the blood-brainbarrier $(10)$.

The highest concentrations of cobalamin per mg protein were contained in the mitochondria-rich fractions among the subcellular fractions, although the total contents of cobalamin were highest in the cytosol. The mitochondrial fraction included some contamination with other intracellular organellae. However, the microsomal fraction which was much purer showed cobalamin contents considerably lower than in the mitochondrial fraction. The larger binder was also obtained in large amounts in the mitochondria-rich fractions, and it was almost saturated with endogenous cobalamin. The elution region of the binder was consistent with those of cobalamin-dependent enzymes. Therefore, part of the larger binder obtained might include these enzymes, though their activities have not yet been studied.

In animals, cobalamin serum levels and its contents in the brain are correlated fairly well (11). In the human brain, the cobalamin contents may be influenced by the serum levels of cobalamin.

The above-mentioned results represent the average values of cobalamin contents in the brains of the elderly. In the young, the biologic aspects of cobalamin are perhaps slightly different from these values.

\section{REFERENCES}

1) Chanarin, I. (1969): The assay and concentration of vitamin $B_{12}$ in serum, liver, and cerebrospinal fluid, in The Megaloblastic Anaemia, F. A. Davis Co., Philadelphia, pp. 192-229.

2) Worm-Petersen, J. (1962): Vitamin $B_{12}$ in the serum and cerebrospinal fluid in neurological diseases. Acta Neurol. Scand., 38, 241-255.

3) Barrows, C. H., and Chow, B. F. (1957): Fractionation of radioactive $B_{12}$-complex in 
kidney homogenates. Proc. Soc. Exp. Biol. Med., 95, 517-520.

4) Strength, D. R., Alexander, W. F., and Wask, J. P. (1959): Intracellular distribution of vitamin $\mathrm{B}_{12}-\mathrm{Co}^{60}$ in liver and kidney of $\mathrm{B}_{12}$ deficient and normal rats. Proc. Soc. Exp. Biol. Med., 102, 15-18.

5) Pletsch, Q., and Coffey, J. W. (1969): Intracellular distributions of vitamin $B_{12}$ in rat liver. Fed. Proc., 28, 628.

6) Whittaker, V. P. (1959): The isolation and characterization of acetyl-choline-containing particles from brain. Biochem. J., 72, 694-706.

7) Linnell, J. C., Hoffbrand, A. V., Hussein, H. A. A., Wise, I. J., and Matthews, D. M. (1974): Tissue distribution of coenzyme and other forms of vitamin,$B_{12}$ in control subjects and patients with pernicious anemia. Clin. Sci. Mol. Biol., 46, 163-172.

8) Worm-Petersen, J. (1964): Vitamin $\mathbf{B}_{12}$, haemoglobin, and iron concentrations in human brain tissue. Acta Neurol. Scand., 40, 241-248.

9) Cooperman, J. M., Luhby, A. L., Teller, D. N., and Marley, J. F. (1960): Distribution of radioactive and non-radioactive vitamin $\mathrm{B}_{12}$ in the dog. J. Biol. Chem., 235, 191194.

10) Katzman, R. (1972): Blood-brain-CSF barriers, in Basic Neurochemistry, ed. by Albers, R. W., Siegel, G. J., Katzman, R., and Agranoff, B. W., Little Brown, Boston, pp. 249263.

11) Dreyfus, P. M. (1970): Biochemical observations on experimental vitamin $B_{12}$ deficiency, Neurology, 20, 402. 